Case Report

\title{
Ramsay Hunt Syndrome in a Patient with Rheumatoid Arthritis after Treatment with Infliximab
}

\author{
Yoshio Nagayama, ${ }^{1}$ Naoki Matsushiro, ${ }^{2}$ Akihide Nampei, ${ }^{1}$ Hideo Hashimoto, ${ }^{3}$ and Kenrin \\ $\mathrm{Shi}^{4}$ \\ ${ }^{1}$ Department of Orthopaedic Surgery, Osaka Rosai Hospital, 1179-3 Nagasonecho, Kita-ku, Sakai 591-8025, Japan \\ ${ }^{2}$ Department of Otolaryngology, Osaka Police Hospital, 10-31 Kitayamacho, Tennoji-ku, Osaka 543-0035, Japan \\ ${ }^{3}$ Rinku Hashimoto Rheumatology Orthopaedics, 1 Rinku Orai kita, Izumisano, Osaka 598-0048, Japan \\ ${ }^{4}$ Department of Orthopaedic Surgery, Osaka University Graduate School of Medicine, 2-2 Yamadaoka, Suita, Osaka, Japan
}

Correspondence should be addressed to Akihide Nampei; ak-nampei@orh.go.jp

Received 4 December 2013; Accepted 24 December 2013; Published 9 February 2014

Academic Editors: R. Cevik, G. O. Littlejohn, J. Mikdashi, L. Stojanovich, and A. Zoli

Copyright (c) 2014 Yoshio Nagayama et al. This is an open access article distributed under the Creative Commons Attribution License, which permits unrestricted use, distribution, and reproduction in any medium, provided the original work is properly cited.

A 39-year-old female patient with rheumatoid arthritis developed Ramsay Hunt syndrome after infliximab treatment. This condition is caused by the reactivation of varicella zoster virus infection in the geniculate ganglion of facial nerve in the host's immunosuppression. She was treated immediately with valaciclovir and hydrocortisone, and the complete recovery was achieved at 6 months after the onset. This is the first report of Ramsay Hunt syndrome as an adverse effect of infliximab in rheumatoid arthritis.

\section{Introduction}

Infliximab (IFX) is the firstly introduced biologic agent for the treatment of rheumatoid arthritis (RA) and its successful efficacy for the patients with methotrexate (MTX) resistant RA has been proven over ten years [1-3]. On the other hand, serious opportunistic infection and reactivation of pathogens, such as tuberculosis, Pneumocystis jiroveci, hepatitis B virus (HBV), cytomegalovirus (CMV), Epstein-Barr virus (EBV), and human immunodeficiency virus, have been reported to be associated with IFX treatment $[4,5]$.

Ramsay Hunt syndrome is characterized by herpetic vesicles in or around the ear, facial nerve paralysis, and vestibulocochlear nerve paralysis manifesting hearing loss and vertigo. It is caused by reactivation of varicella zoster virus (VZV) latently and persistently infected in the geniculate ganglion of facial nerve, often in immunosuppressed conditions of the host. Early intervention is important for complete recovery of the paralysis, but if not, it is more likely to remain permanent damage. Although several reports about VZV reactivation in RA patients have been published, reports on Ramsay Hunt syndrome in RA patients are very few. Here we report a case of Ramsay Hunt syndrome recognized in a patient with RA after treatment with IFX.

\section{Case Report}

A 39-year-old woman, diagnosed as RA in 1997, had been treated with MTX, bucillamine, and prednisolone, but unfortunately was resistant to these medications. In May 2005, her disease activity of RA elevated as high as Disease Activity Score including a 28-joint count (DAS28[6])-erythrocyte sedimentation rate (ESR) of 5.60. Moreover, the progression of joint destruction and functional status was recognized as stage III and class III by Steinbrocker's classification [7, 8], respectively. Then IFX, a biologic agent inhibiting tumor necrosis factor (TNF)- $\alpha$, was introduced with a dose of $3 \mathrm{mg} / \mathrm{kg}$. After three times of IFX administration at 0,2 , and 6 weeks, DAS28-ESR improved significantly to 3.96 .

Ten days after the third infusion of IFX, she felt sore throat, dysphagia, and left otalgia, with development of vesicles on the left pinna. Then three days after these initial symptoms, she felt motor paralysis on the left side of the 


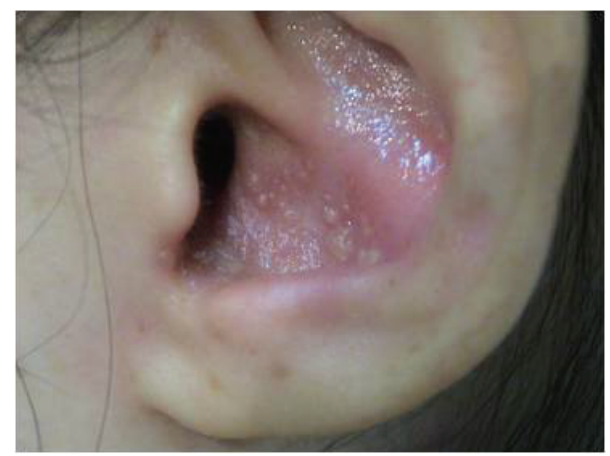

FIGURE 1: Vesicles on the left pinna.

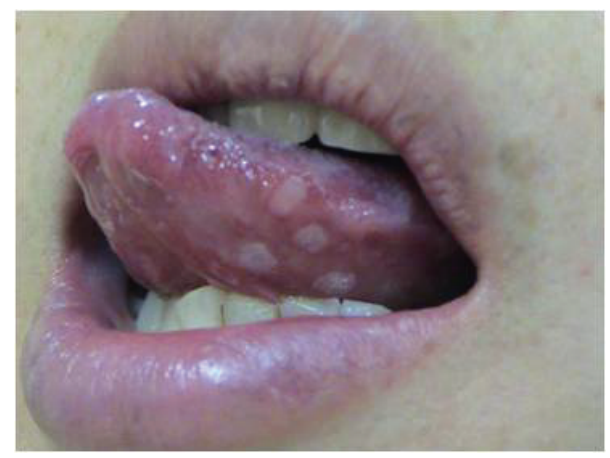

Figure 2: Vesicles on the left side of tongue.

face. She visited otolaryngologist in a private clinic who immediately consulted the otolaryngology department in our hospital at the same day; thereafter, she was diagnosed with Ramsay Hunt syndrome. Physical examination presented multiple vesicles on the left pinna, as well as on the left side of soft palate, tongue, and epiglottic vallecula (Figures 1 and 2). Facial nerve score was 4 points with Yanagihara' grading scale ([9]; total, 40 points). Neurootological examination presented sensorineural hearing loss $(36.3 \mathrm{~dB}$; normal value, $\leq 30 \mathrm{~dB}$ ), nystagmus, and diminished lacrimation (Schirmer test, $7 \mathrm{~mm}$; normal value, $\geq 10 \mathrm{~mm}$ ) on the left side, with the disappearance of the left stapedial reflex and the reduced sense of taste (electrogustometry, $32 \mathrm{~dB}$; normal value, $\leq 8 \mathrm{~dB}$ ). Since such symptoms as sternocleidomastoid muscle atrophy, trapezius muscle paralysis, neck stiffness, and Kernig's sign were not recognized, either accessory nerve paralysis or meningitis was not suggested. Serological study by enzyme immunoassay (EIA) for specific antibodies against VZV presented IgM 0.45 (normal value, $<0.80$ antibody index) and IgG 29.3 (normal value, <2.0 EIA titer). At 12 days after the onset, increased levels of VZV antibodies were recognized: IgM 2.02 and IgG 83.9 (Table 1).

Treatment was started immediately after her visit at our hospital with valaciclovir $3000 \mathrm{mg}$ for 7 days and hydrocortisone sodium succinate for 9 days (500 mg for 3 days, $300 \mathrm{mg}$ for 3 days, and $100 \mathrm{mg}$ for 3 days), while all medications for RA, IFX, MTX, bucillamine, and prednisolone were discontinued. At 6 days after the onset, left recurrent laryngeal nerve
TABLE 1: Serological study of varicella zoster virus (VZV).

\begin{tabular}{lcccc}
\hline & Day 3 & Day 12 & Day 25 & Day 62 \\
\hline IgM (antibody index) & 0.45 & 2.02 & 1.35 & 0.55 \\
IgG (EIA titer) & 29.3 & 83.9 & 68.6 & 47.6 \\
\hline
\end{tabular}

Values of specific antibodies against VZV, IgM, and IgG are shown in a timecourse manner. Antibodies were studied by enzyme immunoassay (EIA). Normal value; IgM: $<0.80$ antibody index; IgG: $<2.0$ EIA titer.

paralysis and left glossopharyngeal nerve paralysis, the symptom of which is characterized as "curtain sign", appeared. At 7 days after the onset, electroneurography (ENoG) and nerve excitability test (NET) were performed for prognostic diagnosis [10], and ENoG was 24.5\% (excellent, ENoG $\geq$ 40\%; good, $20 \% \leq \mathrm{ENoG}<40 \%$; fair, $10 \% \leq \mathrm{ENoG}<20 \%$; poor, ENoG < 10\%), while NET was $4.2 \mathrm{~mA}$ (good, NET $\leq$ $3.5 \mathrm{~mA}$; fair, $3.5 \mathrm{~mA}<\mathrm{NET} \leq 20 \mathrm{~mA}$; poor, NET > $20 \mathrm{~mA}$ ), both indicating unfavorable prognosis of paralysis. Then another course of hydrocortisone sodium succinate with the same dose for 9 days was conducted (Figure 3). After these treatments were introduced, facial nerve paralysis, as graded by Yanagihara's scale, improved successfully to 36 points at 3 months after the onset, and finally to full marks at 6 months (Figure 4). Other cranial nerve symptoms recovered at 2 months after the onset. However, facial synkinesis appeared at 3 months after the onset and remained even at 6 months, though it improved gradually to low grade. As for serological study, levels of VZV antibodies demonstrated a tendency to decrease after the treatment was introduced, and IgM level declined to normal value, 0.55 , at 2 months after the onset whereas IgG level still remained high, 47.6 (Table 1).

With regard to treatment of $\mathrm{RA}$, prednisolone $10 \mathrm{mg} /$ day and bucillamine $300 \mathrm{mg} /$ day were readministered at 26 days after the onset of Ramsay Hunt syndrome. Then MTX $4 \mathrm{mg} /$ week was readministered at 5 months after the onset and was increased to $8 \mathrm{mg} /$ week at 6 months. Disease activity of RA elevated as high as DAS28-ESR of 4.98 at 3 months after the onset but finally improved to DAS28-ESR of 2.68 after readministration of medications, especially of MTX. Recurrence of Ramsay Hunt syndrome was not recognized, and the patient transferred to another hospital for her own reasons at 9 months after the onset (Figure 4).

\section{Discussion}

It is over ten years that biological agents such as IFX have been utilized for RA, and there have been many clinical evidences as well as experiences of their efficacy for MTX resistant RA [1-3], leading to the current general concept that they are indispensable medications for RA treatment [11-14]. However, the development of bacterial, viral, and fungal infections is one of the well-known and sometimes severe adverse events of these medications with potent immunosuppressive effect $[4,5,15,16]$, and reactivation of old or resolved infections such as tuberculosis, HBV, CMV, and EBV has been reported $[6,17-21]$.

Among 5040 RA patients with episodes of herpes zoster in the German biotherapy registry, TNF inhibitors were 
Auricular vesicle

Facial nerve paralysis

Vesicles in the soft palate, tongue, and epiglottic vallecula

Recurrent laryngeal nerve paralysis and glossopharyngeal nerve paralysis

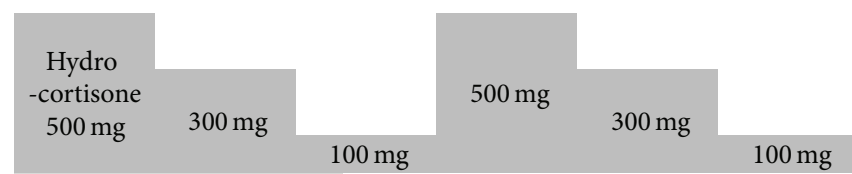

Valaciclovir $3000 \mathrm{mg}$

\begin{tabular}{llll}
\hline 0 & 3 & 10 & 12
\end{tabular}

\section{Day}

FIGURE 3: Initial clinical course of Ramsay Hunt syndrome. At 3 days after the onset, the treatment was started with valaciclovir 3000 mg for 7 days and hydrocortisone sodium succinate for 9 days (500 mg for 3 days, $300 \mathrm{mg}$ for 3 days, and $100 \mathrm{mg}$ for 3 days), followed by another course of hydrocortisone sodium succinate with the same dose for 9 days. Vesicles, recurrent laryngeal nerve paralysis, and glossopharyngeal nerve paralysis were relieved soon after the treatment but facial nerve paralysis continued.

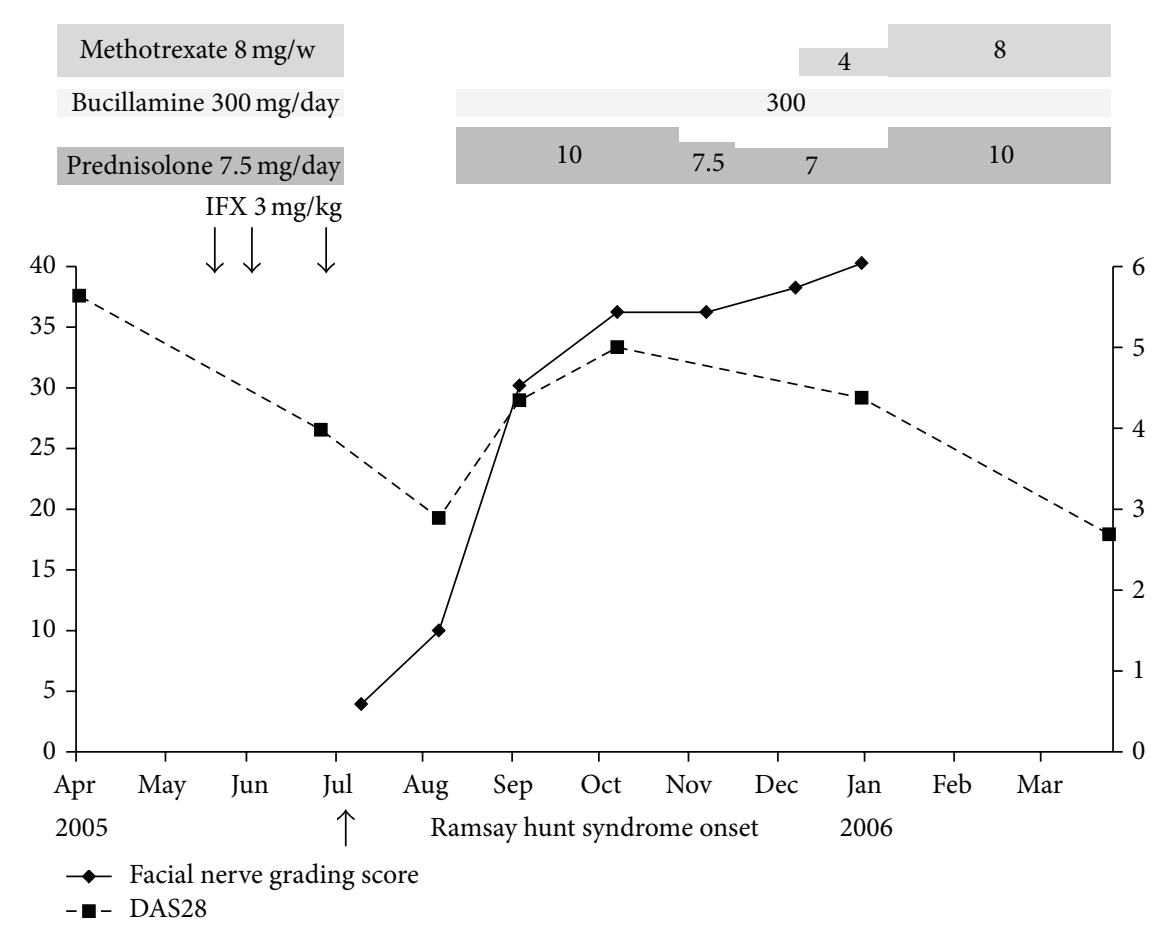

FIGURE 4: Clinical course of facial nerve paralysis and RA. Facial nerve paralysis as graded by Yanagihara's scale recovered completely at 6 months after onset, except for a slight synkinesis. DAS28-ESR elevated after discontinuation of RA treatment but relieved after readministration of medications including MTX. RA: rheumatoid arthritis; DAS28: Disease Activity Score including a 28-joint count; ESR: erythrocyte sedimentation rate; MTX: methotrexate. 
reported to be administered in 3266 patients, among which IFX was reported in 591, as compared to 1774 patients without TNF inhibitors, concluding that TNF inhibitors were significantly associated with herpes zoster (hazards ratio: 2.24) [22]. Also, in a large-scale controlled study of IFX in patients with ulcerative colitis, the incidence of herpes zoster episode increased significantly in the IFX group (1.3\%), as compared to the placebo group (0.4\%) during 54 weeks [23].

Ramsay Hunt syndrome is a rare condition which is caused by reactivation of latent or persistent infection of VZV in the geniculate ganglion of facial nerve, occurring mostly in the host's immunosuppression [24, 25]. Its major symptoms are herpetic vesicles in or around the ear, facial nerve paralysis, and vestibulocochlear nerve paralysis presenting hearing loss and vertigo, and the annual incidence was reported previously as 5/100,000 person-year [26], much less than that of herpes zoster reported as over 300/100,000 person-year [27, 28]. Moreover, there is only one case report of Ramsay Hunt syndrome occurring in a patient with RA under etanercept treatment [29], and there have so far been no reports on Ramsay Hunt syndrome in RA patients after IFX treatment.

As the grade of facial nerve paralysis in Ramsay Hunt syndrome is severe, the prognosis is worse, and early treatment is thought necessary for complete recovery [30]. Among 28 patients in whom the treatment was started within 3 days from the onset of facial nerve paralysis, complete recovery was observed in 21 patients (75\%), while it was observed in 14 (48\%) among 29 patients in whom the treatment was started at 4 to 7 days after the onset. Furthermore, among 23 patients in whom the treatment was started later than 7 days, the complete recovery was only in 7 (30\%) and permanent as well as none or less severe paralysis remained in other patients [31].

In the case presented in this paper, it is assumed that inhibition of TNF- $\alpha$ by IFX promoted the reactivation of latently infected VZV in the geniculate ganglion of facial nerve and caused Ramsay Hunt syndrome. Fortunately, an otolaryngology specialist appropriately diagnosed and the treatment was started as early as at 3 days after the onset, after which the complete recovery of the paralysis was achieved. In conclusion, this is the first report of Ramsay Hunt syndrome as an adverse effect following IFX treatment. Rheumatologists should be aware of this condition, especially for the patients with immunosuppressive treatments. Also, when this condition is suspected, immediate treatment should be introduced after the proper diagnosis by an otolaryngology specialist.

\section{Conflict of Interests}

The authors declare that there is no conflict of interests in the publication of the paper.

\section{Authors' Contribution}

Yoshio Nagayama and Naoki Matsushiro contributed equally to this paper.

\section{References}

[1] P. E. Lipsky, D. M. F. M. van der Heijde, E. W. St. Clair et al., "Infliximab and methotrexate in the treatment of rheumatoid arthritis," The New England Journal of Medicine, vol. 343, no. 22, pp. 1594-1602, 2000.

[2] R. Maini, E. W. St Clair, F. Breedveld et al., "Infliximab (chimeric anti-tumour necrosis factor $\alpha$ monoclonal antibody) versus placebo in rheumatoid arthritis patients receiving concomitant methotrexate: a randomised phase III trial," The Lancet, vol. 354, no. 9194, pp. 1932-1939, 1999.

[3] P. C. Taylor, "Anti-tumor necrosis factor therapies," Current Opinion in Rheumatology, vol. 13, pp. 164-169, 2001.

[4] J. J. Gómez-Reino, L. Carmona, V. Rodríguez Valverde, E. M. Mola, and M. D. Montero, "Treatment of rheumatoid arthritis with tumor necrosis factor inhibitors may predispose to significant increase in tuberculosis risk: a multicenter activesurveillance report," Arthritis and Rheumatism, vol. 48, no. 8, pp. 2122-2127, 2003.

[5] T. Takeuchi, Y. Tatsuki, Y. Nogami et al., "Postmarketing surveillance of the safety profile of infliximab in 5000 Japanese patients with rheumatoid arthritis," Annals of the Rheumatic Diseases, vol. 67, no. 2, pp. 189-194, 2008.

[6] M. L. L. Prevoo, M. A. van 'T Hof, H. H. Kuper, M. A. van Leeuwen, L. B. A. van de Putte, and P. L. C. M. van Riel, "Modified disease activity scores that include twenty-eight-joint counts: development and validation in a prospective longitudinal study of patients with rheumatoid arthritis," Arthritis and Rheumatism, vol. 38, no. 1, pp. 44-48, 1995.

[7] O. Steinbrocker, C. H. Traeger, and R. C. Batterman, “Therapeutic criteria in rheumatoid arthritis," Journal of the American Medical Association, vol. 140, no. 8, pp. 659-662, 1949.

[8] M. C. Hochberg, R. W. Chang, I. Dwosh, S. Lindsey, T. Pincus, and F. Wolfe, "The American College of Rheumatology 1991 revised criteria for the classification of global functional status in rheumatoid arthritis," Arthritis and Rheumatism, vol. 35, no. 5, pp. 498-502, 1992.

[9] Y. Satoh, J. Kanzaki, and S. Yoshihara, "A comparison and conversion table of "the House-Brackmann facial nerve grading system" and "the Yanagihara grading system"', Auris Nasus Larynx, vol. 27, no. 3, pp. 207-212, 2000.

[10] E. Esslen, "Electromyography and electroneurography," in Proceedings of the 3rd International Symposium on Facial Nerve Surgery, Facial Nerve Surgery, U. Fisch, Ed., pp. 93-100, Anstelveen, Zurich, Switzerland, August 1976.

[11] R. Koike, T. Takeuchi, K. Eguchi, and N. Miyasaka, "Update on the Japanese guidelines for the use of infliximab and etanercept in rheumatoid arthritis," Modern Rheumatology, vol. 17, no. 6, pp. 451-458, 2007.

[12] R. Koike, M. Harigai, T. Atsumi et al., "Japan College of Rheumatology 2009 guidelines for the use of tocilizumab, a humanized anti-interleukin-6 receptor monoclonal antibody, in rheumatoid arthritis," Modern Rheumatology, vol. 19, no. 4, pp. 351-357, 2009.

[13] J. S. Smolen, R. Landewé, F. C. Breedveld et al., "EULAR recommendations for the management of rheumatoid arthritis with synthetic and biological disease-modifying antirheumatic drugs," Annals of the Rheumatic Diseases, vol. 69, pp. 964-975, 2010.

[14] J. A. Singh, D. E. Furst, A. Bharat et al., "2012 update of the 2008 American college of rheumatology recommendations for the use of disease-modifying antirheumatic drugs and biologic 
agents in the treatment of rheumatoid arthritis," Arthritis Care and Research, vol. 64, no. 5, pp. 635-639, 2012.

[15] H. Okubo, M. Iwamoto, T. Yoshio et al., "Rapidly aggravated Mycobacterium avium infection in a patient with rheumatoid arthritis treated with infliximab," Modern Rheumatology, vol. 15, no. 1, pp. 62-64, 2005.

[16] S. Mori, F. Imamura, C. Kiyofuji et al., "Pneumocystis jiroveci pneumonia in a patient with rheumatoid arthritis as a complication of treatment with infliximab, anti-tumor necrosis factor $\alpha$ neutralizing antibody," Modern Rheumatology, vol. 16, no. 1, pp. 58-62, 2006.

[17] Y. Hirano, T. Kojima, Y. Kanayama, H. Ishikawa, and N. Ishiguro, "A case of lung tuberculosis in a patient with rheumatoid arthritis treated with infliximab after antituberculosis chemoprophylaxis with isoniazid," Modern Rheumatology, vol. 19, no. 3, pp. 323-328, 2009.

[18] H. Tsuboi, A. Tsujii, A. Nampei et al., "A patient with rheumatoid arthritis treated with tocilizumab together with lamivudine prophylaxis after remission of infliximab-reactivated hepatitis B," Modern Rheumatology, vol. 21, no. 6, pp. 701-705, 2011.

[19] A. Tamori, T. Koike, H. Goto et al., "Prospective study of reactivation of hepatitis $B$ virus in patients with rheumatoid arthritis who received immunosuppressive therapy: evaluation of both HBsAg-positive and HBsAg-negative cohorts," Journal of Gastroenterology, vol. 46, no. 4, pp. 556-564, 2011.

[20] G. Haerter, B. J. Manfras, Y. de Jong-Hesse et al., "Cytomegalovirus retinitis in a patient treated with anti-tumor necrosis factor alpha antibody therapy for rheumatoid arthritis," Clinical Infectious Diseases, vol. 39, no. 9, pp. e88-e94, 2004.

[21] M. Colaci, M. Sebastiani, G. Sandri, M. Meacci, and C. Ferri, "Epstein-barr virus reactivation after infliximab in rheumatoid arthritis: a case report," Case Reports in Infectious Diseases, vol. 2011, Article ID 530568, 3 pages, 2011.

[22] A. Strangfeld, J. Listing, P. Herzer et al., "Risk of herpes zoster in patients with rheumatoid arthritis treated with anti-TNF- $\alpha$ agents," Journal of the American Medical Association, vol. 301, no. 7, pp. 737-744, 2009.

[23] P. Rutgeerts, W. J. Sandborn, B. G. Feagan et al., "Infliximab for induction and maintenance therapy for ulcerative colitis," The New England Journal of Medicine, vol. 353, no. 23, pp. 24622476, 2005.

[24] A. Aviel and G. Marshak, "Ramsay Hunt syndrome: a cranial polyneuropathy," American Journal of Otolaryngology, vol. 3, no. 1, pp. 61-66, 1982.

[25] D. S. Asnis, L. Micic, and D. Giaccio, "Ramsay hunt syndrome presenting as a cranial polyneuropathy," Cutis, vol. 57, no. 6, pp. 421-424, 1996.

[26] K. K. Adour, "Otological complications of herpes zoster," Annals of Neurology, vol. 35, pp. S62-S64, 1994.

[27] R. E. HOPE-SIMPSON, “The nature of herpes zoster," Proceedings of the Royal Society of Medicine, vol. 58, pp. 9-20, 1965.

[28] R. P. Insinga, R. F. Itzler, J. M. Pellissier, P. Saddier, and A. A. Nikas, "The incidence of herpes zoster in a United States administrative database," Journal of General Internal Medicine, vol. 20, no. 8, pp. 748-753, 2005.

[29] D. Kaski, N. Davies, and B. M. Seemungal, "Varicella-zoster virus meningo-rhombencephalitis presenting as Ramsey Hunt," Neurology, vol. 4, pp. 2291-2292, 2012.

[30] C. J. Sweeney and D. H. Gilden, "Ramsay Hunt syndrome," Journal of Neurology Neurosurgery and Psychiatry, vol. 71, no. 2, pp. 149-154, 2001.
[31] S. Murakami, N. Hato, J. Horiuchi, N. Honda, K. Gyo, and N. Yanagihara, "Treatment of Ramsay Hunt syndrome with acyclovir-prednisone: significance of early diagnosis and treatment," Annals of Neurology, vol. 41, no. 3, pp. 353-357, 1997. 


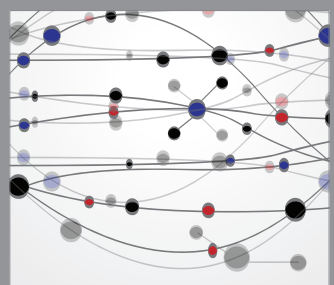

The Scientific World Journal
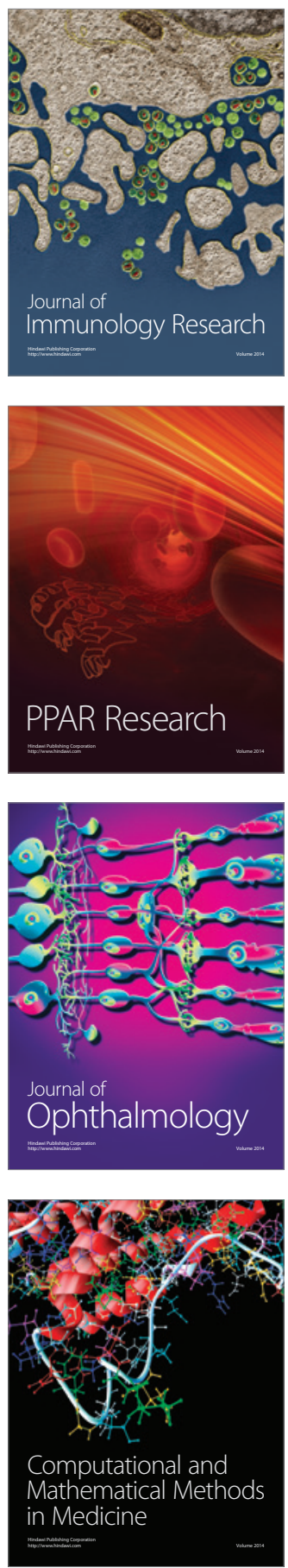

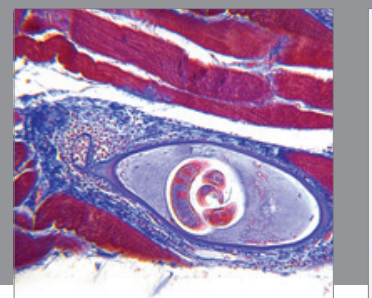

Gastroenterology

Research and Practice
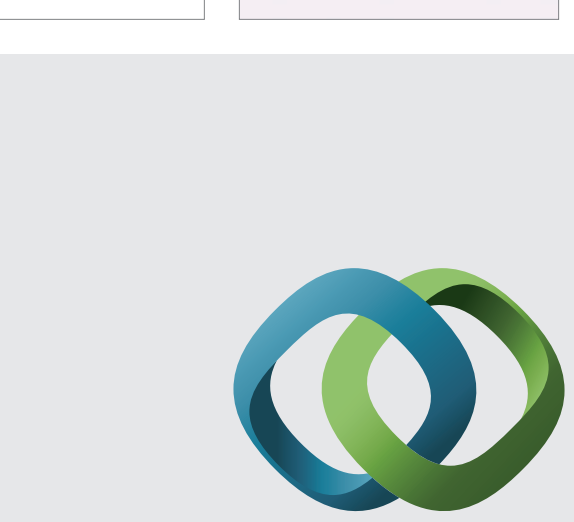

\section{Hindawi}

Submit your manuscripts at

http://www.hindawi.com
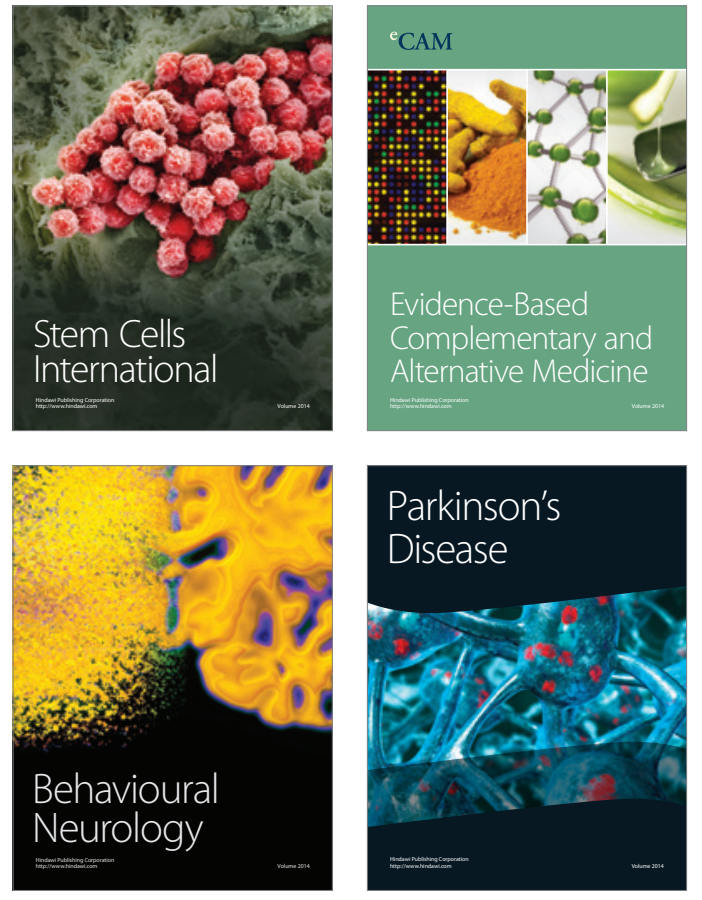
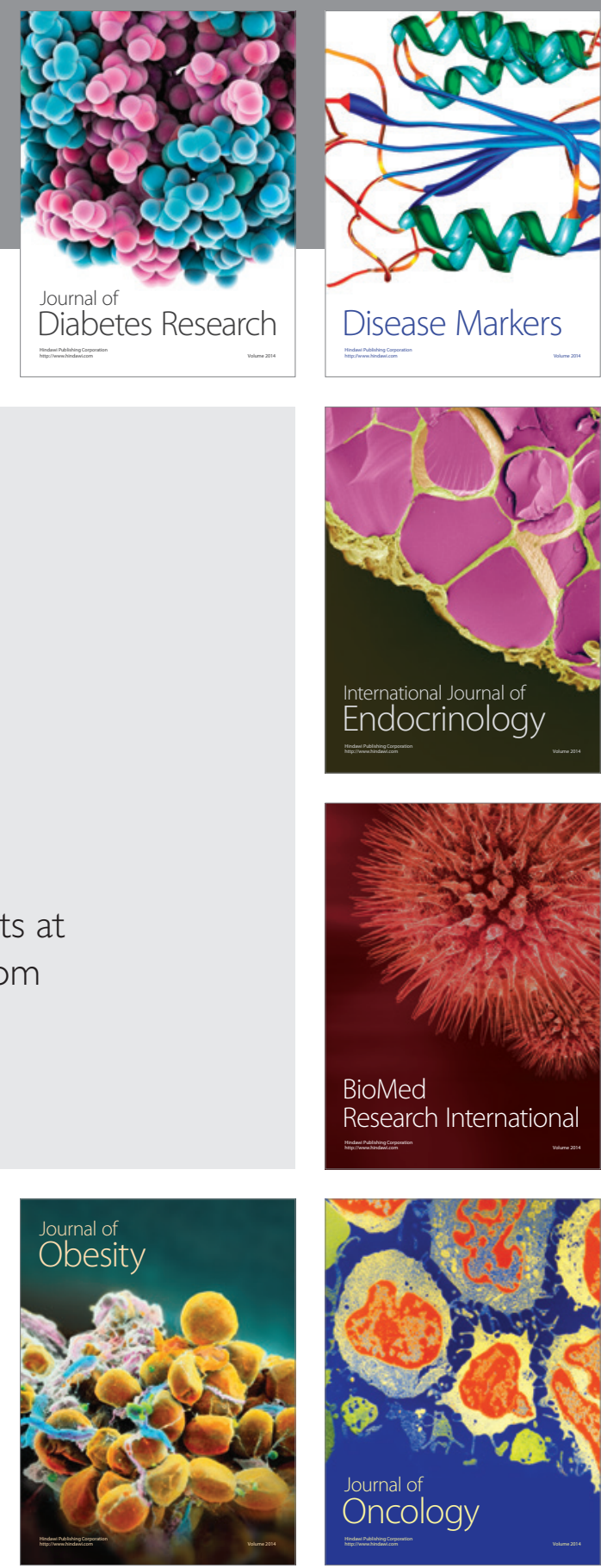

Disease Markers
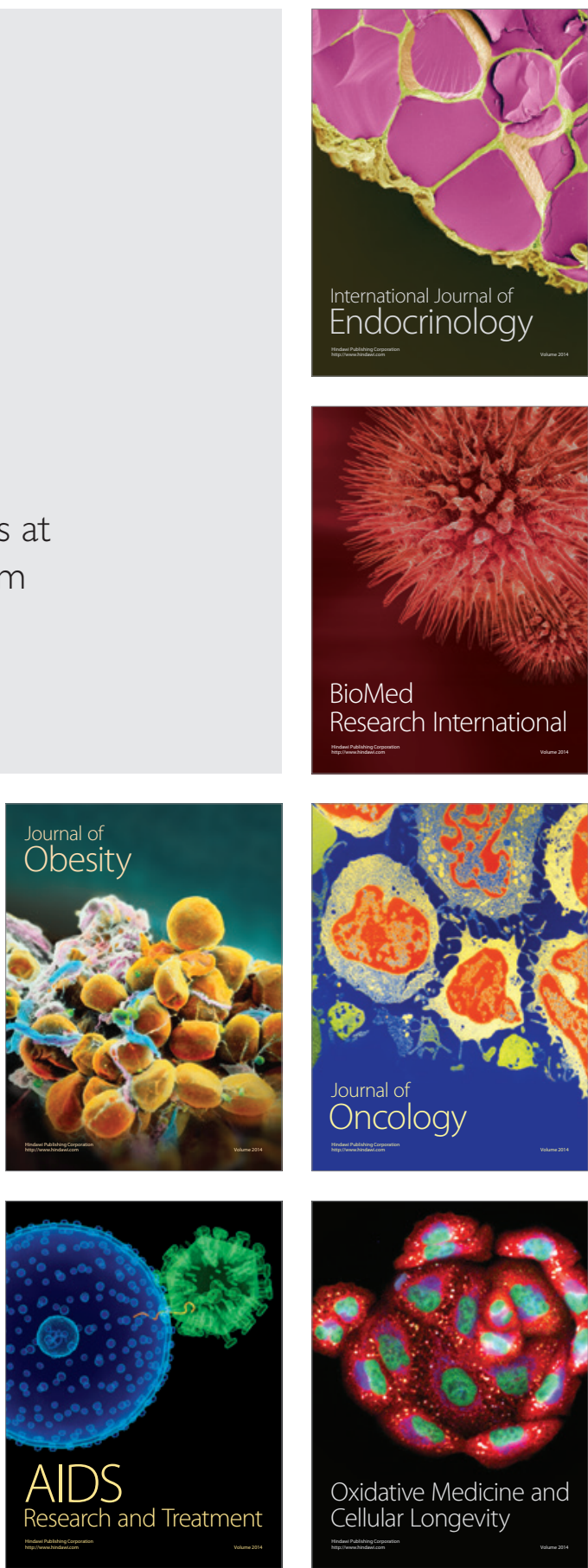